\title{
Turismo y patrones de consumo artesanal en Teotihuacán, México
}

\author{
Verónica Ortega Cabrera \\ vortegac@uawemex.mx \\ Universidad Autónoma del Estado de México, \\ Valle de Teotihuacán \\ México \\ Susana Esquivel Rios \\ sesquivelr@uaemex.mx \\ Universidad Autónoma del Estado de México, \\ Valle de Teotihuacán \\ México \\ Norma Lizbet González Cororna \\ nlgonzalezc@uaemex.mx \\ Universidad Autónoma del Estado de México, \\ Valle de Teotihuacán \\ México
}

\section{RESUMEN}

El presente trabajo se enfoca a describir el vínculo entre el fenómeno turístico y las dinámicas productivas de los artesanos de obsidiana en el valle de Teotihuacán, Estado de México, específicamente en el poblado de San Francisco Mazapa, una comunidad que produce la mayor cantidad de artesanías que se ofrecen a los turistas en la zona arqueológica Patrimonio Mundial.

A través de una metodología cualitativa y una estrategia descriptiva, mostramos las condiciones en que operan las redes artesanales dedicadas a la producción de objetos de obsidiana y la relación que éstas guardan con los principales actores que dinamizan el turismo en esta zona del Estado de México, generando patrones productivos que satisfacen las demandas de un turismo con intereses diversos, entre los que destacan los rasgos de identidad, el esoterismo y el ornato, bajo un discurso en el que se idealiza el pasado prehispánico.

Palabras clave: artesanías de obsidiana; turismo; Teotihuacán 


\title{
Tourism and craft consumption patterns in Teotihuacán, Mexico
}

\begin{abstract}
The present work focuses on describing the link between the tourist phenomenon and the productive dynamics of obsidian artisans in the Teotihuacán Valley, State of Mexico, specifically in the town of San Francisco Mazapa, a community that produces the largest number of handicrafts offered to tourists in the World Heritage archaeological zone. Through a qualitative methodology and a descriptive strategy, we show the conditions in which the craft networks dedicated to the production of obsidian objects operate and the relationship they have with the main actors that stimulate tourism in this area of State of Mexico, generating productive patterns that satisfy the demands of tourism with diverse interests, among which identity traits, esotericism and ornamentation stand out, under a discourse in which the pre-Hispanic past is idealized.
\end{abstract}

Keywords: obsidian handicrafts, tourism, Teotihuacan

Artículo recibido: 02 enero 2022 Aceptado para publicación: 28 enero 2022 Correspondencia: vortegac@uaemex.mx Conflictos de Interés: Ninguna que declarar 


\section{INTRODUCCIÓN}

Hacia 1920, la población del Valle de Teotihuacán se componía en su mayoría de indígenas de habla náhuatl y una de las principales preocupaciones de las políticas gubernamentales del momento era incorporarlos al desarrollo económico y cultural, impulsado por la incipiente industrialización del país. Manuel Gamio promovió la creación de una Escuela de Artes y Oficios, con la que lograría atender dos cuestiones de suma trascendencia: por un lado se instruía a los pobladores indígenas en labores que les permitirían un ingreso económico y por otro se desarrollaban objetos que apuntalaban un concepto de identidad nacional (Gallegos, 1999, p. 234), en el que se fusionaba el carácter indígena con las técnicas europeas de trabajo, bajo dinámicas comerciales capitalistas que darían una amplia circulación a esos objetos que hoy llamamos artesanías.

El centro de artes y oficios desarrollado en el Campamento de las Pirámides de San Juan Teotihuacán contaba con una escuela para la instrucción primaria, así como con varios talleres para la elaboración de objetos de cerámica (talavera), tapetería, obsidiana, arte plumario, carpintería y herrería; además en el Museo Regional -localizado al pie de la Pirámide del Sol-, no solo se exhibían las piezas arqueológicas recuperadas en las excavaciones, sino que también se contaba con una sección en la que se mostraba a los visitantes los objetos producidos en los talleres, de tal forma que parecía existir una continuidad histórica entre lo prehispánico y lo contemporáneo (ídem, p. 235), pues se buscaba que las artesanías contaran con un estilo propio "muy teotihuacano", para posicionarse tanto en el gusto de quienes visitaban las ruinas arqueológicas, como en otros circuitos de venta fuera del valle.

Con el paso de los años, los artesanos especializados en las manufacturas de obsidiana y alfarería son los únicos que han subsistido ante las transformaciones de las actividades económicas en el valle, así como la frecuente migración de los jóvenes hacia los centros urbanos más cercanos. Estas artesanías representan la principal oferta del Valle de Teotihuacán, en donde decenas de talleres producen un importante volumen de objetos que son comercializados principalmente en la zona arqueológica y su perímetro inmediato, que concentran el mayor número de tiendas orientadas al turismo, mismas que funcionan como intermediarias entre los turistas y los artesanos, de tal forma que éstos últimos cumplen el rol de fabricantes en serie para abastecer tanto a los comercios como 
a los más de 400 vendedores ambulantes que, hasta antes de la pandemia de Covid-19, ofrecían las artesanías dentro de la zona arqueológica.

Por lo anterior, nuestro trabajo se enfoca en describir la situación actual de los artesanos teotihuacanos, como un punto de partida que permita posteriores análisis sociales.

\section{MATERIALES Y MÉTODOS}

\subsection{Conceptos}

El concepto de artesanía "engloba las producciones manuales con un valor de uso en la vida doméstica y ritual del campesinado, fundamentalmente indio, que destaca por un plus de tipo estético (un dibujo, un adorno, un acabado) como valor añadido que va de la mano de estilos locales o regionales" (Novelo, 2015, p. 29), por lo cual generalmente se aplica a aquellos objetos de manufactura indígena y campesina, que se expenden principalmente en los sitios turísticos, formando parte de un imaginario colectivo que aporta identidad a un territorio y estableciéndose como referentes de índole geográfico, tradicional, religioso y festivo. Las artesanías tienen protagonismo en las dimensiones económica, cultural e histórica de México, por lo que el discurso sobre ellas ha sido utilizado para muy diversas funciones: generar símbolos, construir imaginarios sociales y convertirse en marca o imagen promocional de una entidad o de la nación (ídem, p. 30). También se asocian con las manufacturas de las clases populares, de hecho, el artesano generalmente es un pequeño productor que desarrolla un trabajo aprendido en el núcleo familiar o comunitario, que se ha hecho de sus herramientas e instrumental de manera paulatina, produciendo fundamentalmente para abastecer mercados locales y/o regionales, en los que deben competir con intermediarios del mercado turístico o adaptarse a un pago muy reducido por sus productos (Paré, 2015, p. 497).

Con el crecimiento de la industria turística, la venta de artesanías tomó una relevancia nunca antes vista, particularmente con las nuevas tendencias de diversificación de los destinos, que delimitaron un segmento relacionado con el patrimonio cultural tangible e intangible denominado turismo cultural, el cual provocó un nuevo tipo de consumo de las manufacturas locales, transformándolas en los ya mencionados souvenirs artesanales. Bajo esta lógica comercial, la producción de artesanías incluyó centros de producción, canales de distribución y puntos de venta, generando una serie de productos en función de la demanda (véase Le Mür, 2015, p. 517), razón por la cual los artesanos han tenido que modificar sus productos de acuerdo con las preferencias y gustos de los clientes, 
trabajando bajo "pedidos" y en muchos casos, incluso, con indicaciones en cuanto al material, las formas y otras características para satisfacer ciertos requisitos, entre los que sobresalen la autenticidad, el exotismo, la portabilidad y el bajo costo, principalmente.

Todo lo anterior se encuentra ligado al proceso productivo y los canales de distribución de los objetos terminados, los cuales requieren de una organización bien estructurada para transformar las materias primas en tiempos y espacios específicos, con el fin de abastecer una serie de necesidades vinculadas a los diversos segmentos de turistas que llegan a Teotihuacán: estudiantes y familias mexicanas, viajeros internacionales, excursionistas juveniles, parejas y grupos de amigos, principalmente; cada uno de los cuales manifiesta preferencias por determinados objetos.

Además, es importante reconocer las principales materias primas utilizadas, así como los materiales adicionales que son incorporados para llegar al producto final con ciertos niveles de calidad, lo que les permitirá posicionarse en circuitos de comercialización de diverso alcance.

Ya hemos señalado líneas arriba la diversidad de las artesanías de acuerdo con la aceptación que éstas tienen entre los turistas, por lo que algunas pueden ser de poco, mediano o alto consumo, mientras que otras son piezas únicas, pero todas cuentan con elementos identitarios con una importante carga cultural, estética y simbólica, debido a la gran proporción de mano de obra que incluyen, pues cada pieza proviene de un taller en el que el artesano tuvo contacto directo con la materia prima y la transformó sin la mediación de grandes recursos tecnológicos ni industriales

\subsection{Método}

Nos proponemos describir el proceso de producción artesanal de objetos de obsidiana en San Francisco Mazapa, Teotihuacán, para identificar patrones de consumo por parte del turismo que visita la zona arqueológica Patrimonio Mundial. Por lo tanto, el alcance de este estudio es descriptivo, toda vez que se establecerán las características del trabajo artesanal de la obsidiana en el Valle de Teotihuacán, identificando las variables que el turismo genera para que esta actividad tenga presencia, relevancia y se adapte a las condiciones económico sociales. De acuerdo con Rojas (2015, p. 7) este tipo de investigación exhibe el conocimiento de la realidad tal como se presenta en una situación de espacio y de tiempo dado; "Se observa y se registra, o se pregunta y se registra. Describe el fenómeno sin introducir modificaciones: tal cual." Y es que si algo falta para 
avanzar en los estudios turísticos de esta región es la descripción del estado de las cosas; a la fecha son escasos los trabajos académicos que den cuenta de las condiciones en que se desarrollan las actividades ligadas al turismo, incluso el fenómeno turístico no ha sido abordado con suficiente solvencia como para dar paso a estudios de tipo analítico.

Retomando los conceptos de Sancho (2001), buscamos identificar al colectivo de individuos que conforma el grupo artesanal de obsidiana en San Francisco Mazapa, así como las variables internas y externas que inciden en su actividad. La estrategia de recolección de datos se aplicó en un contexto empírico real, representado por los artesanos ya mencionados durante el año 2020, seleccionando una muestra de 10 artesanos, que constituyen el $20 \%$ de la población censada en la comunidad; indagando la influencia y los valores a través de los cuales se manifiesta la variable "demanda turística de artesanías".

La estrategia de campo para la recolección de datos primarios fue la entrevista, aplicando un instrumento de recolección de datos de tipo cuestionario estructurado, en atención a los siguientes aspectos: condiciones de trabajo, redes de abastecimiento de materias primas, circuitos de venta de objetos artesanales, características identitarias de las piezas y consumo turístico

\section{RESULTADOS Y DISCUSIÓN}

Los resultados obtenidos durante el trabajo de campo se desglosan en los ocho apartados que presentamos a continuación:

1) Contexto: El poblado de San Francisco Mazapa pertenece al municipio de Teotihuacán, Estado de México; colinda hacia el norte con el municipio de San Martín de las Pirámides, hacia el oeste con la Zona Arqueológica de Teotihuacán, hacia el sur con Santa María Coatlán y hacia el este con la carretera México-Tulancingo y el pueblo de Cuautlacingo, perteneciente al municipio de Otumba. Además de su actividad artesanal, San Francisco Mazapa cuenta con espacios de hospedaje y restaurantes especializados en la atención a los turistas, así como también se han habilitado globo puertos ante la creciente demanda de vuelos en globo aerostático. Durante el período de la pandemia de Covid-19 y ante la imposibilidad de vender sus productos en la zona arqueológica, diversos talleres han optado por abrir sus puertas para la venta directa. 
El gremio artesanal se ha organizado en dos grandes agrupaciones: la "Unión de Artesanos no Asalariados dentro de la Zona Arqueológica de Teotihuacán" y la "Unión de Artesanos Auténticos del Valle de Teotihuacán” (Flores, 2015, p. 27-28).

2) Antecedentes del trabajo artesanal en San Francisco Mazapa: El primer taller de objetos artesanales de obsidiana se fundó hacia 1920, perteneciente a la familia Bazán, que era muy cercana a Manuel Gamio y que se capacitó en la Escuela de Artes y Oficios (Martínez, 2011). La mencionada escuela fue el primer impulso a la actividad artesanal de talla de obsidiana, y después de su cierre no se ha registrado ninguna otra iniciativa para la capacitación formal de los artesanos, de tal forma que el conocimiento de las técnicas de trabajo se transmite de forma empírica, en el entorno familiar o, en algunos casos, las empresas de venta de artesanías capacitan a algunos trabajadores para que elaboren las piezas que ellos comercializan (véase Flores, 2015).

La mayoría de las personas dedicadas a esta actividad se integran a ella desde muy jóvenes, de tal manera que resulta bastante común que, después de algunos años en los que formaron parte de algún taller y han adquirido conocimientos sobre todo el proceso productivo, tomen la iniciativa de comenzar con su propia microempresa, pues también ya conocen los contactos a los cuales pueden recurrir en caso de necesitar crédito, herramienta y ayuda en general. Así, el 90\% de los talleres son resultado del esfuerzo e iniciativa individual en donde el principal obstáculo es la falta de financiamientos y asesorías (ídem). Cabe mencionar que durante el proceso de integración de los municipios del Valle de Teotihuacán al Programa Estatal Pueblos con Encanto del Bicentenario, así como durante la búsqueda del nombramiento de Pueblos Mágicos para Teotihuacán y San Martín de las Pirámides (Ortega, et al., 2020, p. 93435 y 93437) se orientaron diversos financiamientos a algunos talleres por parte del Fondo Nacional para las Artesanías (FONART), así como el estímulo por parte del Instituto de Investigación y Fomento de las Artesanías del Estado de México (IIFAEM) para su credencialización, con lo que algunos artesanos podrían optar a programas de capacitación, promoción, concursos, salud ocupacional, proyectos artesanales estratégicos y comercialización dentro de ferias y exposiciones (Municipio de Teotihuacán, 2017, p. 17), aunque muchos de los entrevistados refieren que todo lo anterior depende de filiaciones partidistas y cercanía de las agrupaciones con los actores políticos. 
Entre los principales retos que afrontan los artesanos se encuentra el incremento en los costos de las materias primas y la presencia cada vez mayor de productos orientales ("chinos") que generan una competencia desleal, al venderse a precios mucho más bajos que los de las artesanías locales, por lo que cada vez un mayor número de artesanos incentivan a sus hijos para que acudan a escuelas de nivel superior, con la finalidad de contar en el taller con personas mejor calificadas, no necesariamente en el arte, sino en aspectos del negocio como la administración, organización, publicidad y ventas. Así, los nuevos artesanos tendrán un nivel educativo más alto que las viejas generaciones en las ramas administrativa, contable, de emprendimiento y comercialización, con lo que se logrará incrementar el capital humano, lo cual les ayudará a buscar innovaciones para mantenerse en el mercado y no abandonar esta valiosa actividad.

3) Base de datos de los talleres registrados: Como ya se mencionó los instrumentos de recolección de datos fueron aplicados en diez talleres dentro del poblado, identificados a través del recorrido directo en la comunidad y por recomendación entre los propios artesanos, quienes ayudaron a establecer el contacto con sus conocidos.

4) Materias primas: La obsidiana es un vidrio volcánico que se forma cuando las lavas incandescentes, a $600{ }^{\circ} \mathrm{C}$, con alto contenido de sílice y aluminio se enfrían rápidamente (Pastrana, 2010, p. 49), y es el material natural más eficiente para la elaboración de instrumentos de corte y penetración. Nuestro país cuenta con diversos yacimientos de obsidiana, pero la que se utiliza en San Francisco Mazapa proviene esencialmente de tres lugares: Jalisco, Hidalgo y en menor medida Otumba, llegando a los talleres básicamente de dos maneras: directamente de las minas a las que acuden los artesanos para adquirirla, o a través de los proveedores que llegan a ofrecerla a la comunidad. Las variedades más utilizadas son la arcoiris, negra, meca (rojiza), café, dorada y gris; el costo promedio de una tonelada de obsidiana negra es de $\$ 50,000.00$ (cincuenta mil pesos), se entrega en bloques que van desde los $100 \mathrm{~kg}$ hasta varias toneladas y es muy importante que el artesano tenga habilidad para seleccionarlos ya que, ocasionalmente, algunos traen fisuras o grietas que reducen la posibilidad de aprovechar todo el material. Por otro lado, se pudo registrar que la obsidiana dorada proviene de las minas de Nopalillo, en la Sierra de las Navajas, Hidalgo, mientras que las variedades roja, negra y arcoiris son extraídas en minas de los municipios de Magdalena y Tequila, Jalisco. 
5) Herramientas de trabajo utilizadas en los talleres: Cortadoras industriales para desbastar los grandes bloques, esmeriles con punta de diamante para dar forma y detallar las figuras, rectificadores o moto-tool para pulir las piezas, tortur para la incrustación de otras piedras como el cuarzo, y lijas para darle brillo y acabado liso a las piezas.

6) Tipos de objetos elaborados: Se pudo registrar que existen especializaciones en ciertos objetos, pues no necesariamente todos los artesanos elaboran piezas completas, ya que algunos grupos maquilan cuentas para ser engarzadas y conformar bisutería, mientras que otros dan terminados especiales como las incrustraciones; un segmento más se dedica al diseño de piezas por pedido de las grandes tiendas ubicadas junto a la zona arqueológica. Los artesanos con menor capacidad adquisitiva compran los remanentes de desbaste de los talleres más grandes, para elaborar principalmente cuentas y aplicaciones para otros objetos como la bisutería. Las cuentas redondas para formar collares, aretes y pulseras son elaboradas principalmente por mujeres, pues a decir de algunos "ellas tienen más delicadeza para ese tipo de piezas".

7) Piezas con mayor demanda: De acuerdo con el análisis de los instrumentos aplicados se presenta el listado de objetos de obsidiana con mayor demanda en orden decreciente: jabones para masaje, tortugas de la buena suerte, "indios" o "muñecos", esferas, pirámides, cráneos y corazones. Otra categoría de artesanías es la de las máscaras teotihuacanas con incrustaciones de concha, turquesa, malaquita, azurita, ojo de tigre así como plata y oro (para pedidos especiales), reproducciones de piezas arqueológicas, mismas que se elaboran principalmente en los talleres vinculados a las grandes tiendas que se ubican en torno a la zona arqueológica. La categoría más reducida es la de hachas, cuchillos y puntas de flecha elaboradas a través de la técnica de percusión, pues requieren de un trabajo muy detallado que debe complementarse con la elaboración de mangos o bastones de madera, pasta epóxica y plásticos.

8) Diseño de un esquema de comercialización de las piezas artesanales de obsidiana a nivel local, regional y panregional: Lo anterior para comprender el impacto de esta producción para el mercado de Teotihuacán así como de otros puntos turísticos en el país, pues Teotihuacán es considerado como el principal centro productor y distribuidor de artesanías de obsidiana a nivel nacional. Si esta actividad ha mantenido una tasa sostenida de crecimiento a lo largo de las últimas cinco décadas, se debe en gran medida a la demanda de los objetos en los circuitos artesanales para el turismo, no solo del valle, sino 
de otros destinos turísticos como la Ciudad de México, Cancún, la Riviera Maya, Los Cabos, Huatulco, Oaxaca y Chiapas. Lo anterior permite explorar una línea de estudio en la que el turismo como práctica local, ha dado pie al desarrollo de cadenas de distribución semejantes a las que la antigua sociedad teotihuacana dominó hace 1500 años, justamente con la misma materia prima, aunque por supuesto, con otra finalidad.

\section{CONCLUSIONES}

Hemos avanzado en una primera fase en la que observamos un panorama con actores sociales que han sido moldeados por la actividad turística desde hace un siglo, y que continúan inmersos en la dinámica marcada por los grandes proyectos de crecimiento en este sector. De hecho, la actividad artesanal existe en Teotihuacán debido al turismo, es su razón de ser y su objetivo final, incluso ampliando su radio de distribución a zonas geográficas lejanas, gracias al prestigio que han adquirido muchos de los artesanos y a la constante innovación, lo que demuestra una buena adaptación a las exigencias del mercado. La siguiente tabla resume la información proporcionada por los artesanos:

Tabla 1. Objetos artesanales producidos en San Francisco Mazapa, Teotihuacán, México

\begin{tabular}{|c|c|c|c|c|}
\hline Formas & $\begin{array}{c}\text { Motivo o } \\
\text { inspiración }\end{array}$ & Usos & $\begin{array}{l}\text { Nivel de } \\
\text { Consumo }\end{array}$ & $\begin{array}{c}\text { Tipos de } \\
\text { obsidiana }\end{array}$ \\
\hline Máscara Teotihuacana & \multirow{11}{*}{ Prehispánico } & \multirow{11}{*}{ Ornato } & \multirow{11}{*}{ Medio } & \multirow{11}{*}{$\begin{array}{l}\text { Negra, dorada } \\
\text { arcoiris, plateada } \\
\text { meca. } \\
\text { Incrustaciones de } \\
\text { plata, concha y } \\
\text { otras piedras }\end{array}$} \\
\hline Cabeza de Quetzalcóatl & & & & \\
\hline Huehuetéotl & & & & \\
\hline $\begin{array}{l}\text { Cabeza de la vida y la } \\
\text { muerte }\end{array}$ & & & & \\
\hline Vasija de mono & & & & \\
\hline Calendario azteca & & & & \\
\hline Cabeza maya & & & & \\
\hline Pirámides escalonadas & & & & \\
\hline Pirámides lisas & & & & \\
\hline Caballeros tigre & & & & \\
\hline ídolos, muñecos, indios & & & & \\
\hline
\end{tabular}




\begin{tabular}{|c|c|c|c|c|}
\hline Jabones para masaje & \multirow{4}{*}{ Contemporáneo } & \multirow{4}{*}{$\begin{array}{l}\text { Utilitario } \\
\text { (spa, } \\
\text { relajación, } \\
\text { terapéutico) }\end{array}$} & \multirow{4}{*}{ Alto } & \multirow{4}{*}{ Negra y dorada } \\
\hline Esferas & & & & \\
\hline Espejos & & & & \\
\hline Gotas & & & & \\
\hline Tortugas & \multirow{6}{*}{ Contemporáneo } & \multirow{6}{*}{$\mid \begin{array}{ll}\text { Ornato } & \mathrm{y} \\
\text { utilitario } & \end{array}$} & \multirow{6}{*}{ Alto } & \multirow{6}{*}{$\begin{array}{l}\text { Negra, dorada, } \\
\text { arcoiris, meca, } \\
\text { incrustaciones de } \\
\text { concha y plata }\end{array}$} \\
\hline Elefantes & & & & \\
\hline Huevos & & & & \\
\hline Corazones & & & & \\
\hline Cuentas & & & & \\
\hline Dijes & & & & \\
\hline Cuchillos & \multirow{4}{*}{ Prehispánico } & \multirow{4}{*}{ Ornato } & \multirow{4}{*}{ Bajo } & \multirow{4}{*}{$\begin{array}{lll}\text { Negra, verde y } & \\
\text { dorada } & & \end{array}$} \\
\hline Hachas & & & & \\
\hline Puntas de flecha & & & & \\
\hline Navajillas & & & & \\
\hline
\end{tabular}

Fuente: Elaboración propia.

En lo que se refiere a los componentes del proceso productivo de los objetos artesanales, es posible visualizar una diversificación que permite el énfasis tanto en los procesos como en la repetición y la personalización, lo que indica que esta actividad es sumamente adaptable a los tiempos y a las exigencias del turismo, manteniendo una vigencia que se vincula en buena medida con el valor simbólico que se le asigna a la obsidiana, ya que por un lado se le considera uno de los principales referentes de la tecnología prehispánica, mientras que por el otro es un material con una fuerte carga esotérica y estética, adecuada para la elaboración de piezas de joyería y objetos terapéuticos, que pueden ser directamente vinculados con el discurso metafísico que muchos turistas buscan en las zonas arqueológicas. Debido a lo anterior, la elaboración de jabones para masaje, esferas, espejos y gotas ha manifestado un aumento considerable en la última década, debido a la 
demanda por parte de turistas que visitan el sitio y que acuden a hoteles con spa, temazcales y espacios en los que se ofrecen terapias alternativas.

Las artesanías inspiradas en piezas arqueológicas mantienen una buena presencia, pero se comercializan en circuitos más especializados pues generalmente sus costos son altos y atienden a la demanda de turistas más informados, generalmente extranjeros, que además del elemento estético buscan rasgos de autenticidad y originalidad. Varios artesanos comentaron acerca de la importancia de que se realicen actividades de capacitación e información acerca de los nuevos hallazgos arqueológicos y la cosmovisión prehispánica, para poder elaborar artesanías con mayor significado.

El caso de Teotihuacán puede ser paradigmático para comprender el desarrollo del turismo cultural; describirlo será además una buena oportunidad para los interesados en los estudios de caso y dotará de información empírica a futuros investigadores, pues hasta el momento se carece de información sistematizada proveniente de un estudio turístico, ya que prevalecen en la zona los enfoques antropológicos, sociológicos, históricos y educativos, pero hay una fuerte carencia de trabajos desde la óptica de la disciplina turística.

\section{CONSIDERACIONES FINALES}

Las artesanías teotihuacanas de obsidiana son elaboradas principalmente por habitantes de los alrededores de la Zona Arqueológica de Teotihuacán, quienes reproducen formas y materiales completamente ajenos a su vida cotidiana, que evocan desde las deidades prehispánicas hasta la imaginería propia de la cultura contemporánea, que incluye figuras de elefantes, tortugas, espejos, esferas, piedras para masaje, corazones, pirámides egipcias y una gran cantidad de elementos que demandan los turistas. De esta forma la artesanía de obsidiana sigue tendencias comerciales, aunque sobresalen algunos artesanos fieles a la tradición de la talla de motivos prehispánicos, que replican piezas arqueológicas famosas, muchas de ellas de origen maya o mexica.

Podemos ligar lo anterior con el hecho de que los artesanos en Teotihuacán no mantienen un contacto directo con los turistas, pues en su mayoría producen en talleres domésticos dentro de las comunidades, para abastecer a los intermediarios (vendedores ambulantes o locatarios) que se encuentran en los alrededores o dentro de la zona arqueológica (Flores, 2015 , p. 40), de tal forma que el turista o el visitante no es consiente del proceso productivo y de las implicaciones culturales del mismo; por su parte muchos artesanos 
asumen un papel de maquiladores, con capacidad para suministrar las formas y diseños que más se comercializan. Por lo tanto, estamos frente a una industria de obsidiana que sigue una línea estética marcada por los hallazgos arqueológicos y los diseños propios de una cultura contemporánea globalizada, que fusiona diversos estilos conforme tienen éxito comercial.

\section{LISTA DE REFERENCIAS}

Bustos F., C. (2009). La producción artesanal. Visión Gerencial, Año 8 No. 1, pp. 37-52. de Melo, C., y Ciliane C. (2015). El souvenir artesanal y la promoción de la imagen del lugar turístico. Estudios y Perspectivas del Turismo, Vol. 24, No. 2, pp. 188-204.

Flores G., J. (2015). Actores, redes socioeconómicas y cadenas productivas en la industria artesanal de la obsidiana en San Martín de las Pirámides y Teotihuacán de Arista, Estado de México. [Tesis de Licenciatura en Geografía Humana, Universidad Autónoma Metropolitana, Unidad Iztapalapa]. Repositorio Institucional.

Gallegos Téllez Rojo, J.R. (1999). La artesanía un modelos social y tecnológico para los indígenas. Política y Cultura, 12, pp. 223-241.

Gudiño, A. (2017). Impulsan las artesanías con la Feria de la Obsidiana. Milenio Diario. https://www.milenio.com/cultura/impulsan-las-artesanias-con-la-feria-de-laobsidiana

Jácome, J. (2015). El impulso del turismo a través de las prácticas asociacionistas. El caso del corredor turístico del Valle de Teotihuacán. Revista de arquitectura, urbanismo y territorios del Instituto de Ciencias Sociales y Humanidades, Benemérita Universidad Autónoma de Puebla, pp. 109-139.

Rivera, R. y Peralta, A. (2016). Turismo Cultural en México. Revista Reporte Anáhuac de Investigación Turística. Año 1, 4, pp. 4-23.

Lagunas A., D. (2010). El dilema de Mata Ortiz. Turismo, artesanía y goce estético en el desierto de Chihuahua, México. Revista Líder, Vol. 17, año 12, pp. 177-201.

Le Mür, R. (2015). El encuentro cultural entre turistas y comunidades indígenas. En Pérez R., S. (Editor) Artesanías y saberes tradicionales, Tomo I, El Colegio de Michoacán, México, pp. 503-525.

Feinman, G. y Nicholas, L. (2006). La producción artesanal en Oaxaca. Rev. Arqueología Mexicana, Vol. XIV, 80, pp.36-43. 
Hernández P., D. (2013). Por un desarrollo artesanal integral. En Sales H., F.J. (Compilador), Las artesanías en México: situación actual y retos, Centro de Estudios Sociales y de Opinión Pública, México, pp. 17-30.

Mercado L., E. (2016). Patrimonio cultural y turismo en el México posrevolucionario. PASOS. Revista de Turismo y Patrimonio Cultural. Vol. 14, 4, pp. 1027-1040, http://ucsj.redalyc.org/articulo.oa? $\mathrm{id}=88146706016$

Martínez C., P. X. (2011). Procesos de aprendizajes artesanales de las personas adultas con la piedra obsidiana; Santa María Coatlán, Teotihuacán. [Tesis de Licenciatura en Educación de Adultos, Universidad Pedagógica Nacional, México). Repositorio Institucional.

Municipio de Teotihuacán (2016) Gaceta Municipal Teotihuacán. El informe de todos. https://www.ipomex.org.mx/recursos/ipo/files_ipo/2016/28/9/28cf94c28378911 1e4736b93ae99cb8d.pdf.

Novelo O., V. (2015). De eso que llamamos artesanías mexicanas. En Pérez R., S. (Editor) Artesanías y saberes tradicionales, Tomo I, El Colegio de Michoacán, México, pp. 29-46.

Ortega C., V., González C., N. L. y Esquivel R., S. (2020). Turismo, patrimonio cultural, sustentabilidad y el megaproyecto del NAIM, una perspectiva desde el Valle de Teotihuacán, México. Brazilian Journal of Development, Curitiba, Vol. 6, No. 11, pp.93429-93443.

Paré O., L. (2015). Artesanía, mercado y sustentabilidad. En Pérez R., S. (Editor) Artesanías y saberes tradicionales, Tomo I, El Colegio de Michoacán, México, pp. 497-502.

Pastrana, A. (2010). La Obsidiana en Mesoamérica. Revista Arqueología Mexicana, 80, pp. 49-54.

Petit-Laurent C., C. A. (2013). Identidad cultural en el objeto y diseño de souvenirs. El caso de Chiloé. [Tesis Doctoral en Bellas Artes, Universidad Complutense de Madrid]. Repositorio Institucional.

Rojas C., M. (2015). Tipos de investigación científica. Una simplificación de la complicada incoherente nomenclatura y clasificación. REDVET Revista Electrónica de Veterinaria, vol. 16, núm. 1, pp. 1-14. 
Ortega Cabrera y otros...

Sancho P., A., et al. (2001). Apuntes de Metodología de la Investigación en Turismo, Organización Mundial del Turismo, Madrid. 